\title{
OBSERVATIONS ON INTERSTITIAL CYSTITIS
}

\author{
JACK LAPIDES, M.D.
}

From the Section of Urology, Department of Surgery, University of Michigan Medical Center, Ann Arbor, Michigan

\begin{abstract}
Interstitial cystitis is more common in young women than previously believed. Findings typical of the disease are a history of diurnal and nocturnal pollakiuria, pelvic discomfort increased with bladder distention, marked bladder tenderness on gentle palpation of the anterior lateral vaginal fornices, less than normal bladder capacity, and a negative urinalysis except for many epithelial cells.
\end{abstract}

A review of the literature relating to Hunner's ulcer discloses that the disease occurs primarily in middle-aged women and is manifested on cystoscopic examination by ulcers and stellate scars which bleed on vesical distention. ${ }^{1-3}$ Typically the patient is noted to complain of round-theclock pollakiuria and pelvic pain or pressure which appears with bladder distention and is relieved on voiding.

The physical examination is normal or nondiagnostic; occasionally there may be suprapubic tenderness. In most cases urinalysis is reported to show no infection but will reveal, on occasion, microscopic hematuria or minimal pyuria.

During the past seven years we have had the opportunity of observing a group of young women, varying from nineteen to twenty-three years of age, who had been treated for increased frequency of urination and bladder discomfort, and who had been diagnosed as having recurrent urinary infection, neurogenic bladder, urethral polyps, urethral stenosis, irritable bladder, psychoneurosis, and others. The patients were referred to our medical center because their symptoms persisted despite a variety of therapeutic regimens, and both they and their physicians desired further consultation.

\section{Method and Materials}

In addition to the usual completc history, physical examination, routine laboratory studies, and urinalysis, each patient was subjected to ex- cretory urography, cystometry, endoscopy, and biopsy of bladder wall. The women were seen at regular intervals as outpatients, and some were admitted to the hospital for therapy as indicated.

The symptoms common to all included increased frequency of diurnal and nocturnal micturition, and perineal and/or suprapubic discomfort on bladder distention with varying degrees of relief on vesical emptying.

Physical examination was essentially negative except for unusual tenderness of the floor of the bladder to touch by the examiner's index finger inserted vaginally and directed laterally to the urethra in the region of the anterior lateral fornices. This finding was present in every patient and varied in intensity depending on the condition of the bladder at the time of examination. The tenderness was as exquisite as that observed in patients with prostatic abscess and acute pollakiuria and perineal pain. When the individual's symptoms were minimal, bladder tenderness was minimal.

All of the excretory urograms showed normal findings except for bladders which appeared to have less than average capacity. Cystometry demonstrated essentially normal innervation and function with the exception of decreased bladder capacity ranging from 175 to $250 \mathrm{ml}$. The small bladder volumes were real and not due to pain, as confirmed under anesthesia.

No obvious ulceration or scarring of the bladder wall was seen on cystoscopic examination. 
There was, however, injection of blood vessels throughout the bladder with varying degrees of diffuse bleeding on distention. Biopsy reports of the bladder wall revealed nonspecific chronic inflammation.

\section{Comment}

The purpose of this report is to emphasize that interstitial cystitis probably occurs as frequently in teenage females and in the third decade of life as it does in menopausal women, and that it may not be associated with ulcers, stellate scars, punctate bleeding on bladder distention, or markedly small-capacity bladders. In view of the lack of so-called typical findings in these girls and young women, it is easy to comprehend the reason for their presentation as diagnostic problems. Our observations suggest a need for reorientation of present concepts regarding interstitial cystitis so that some of the frustrations of the patient and her physician can be alleviated. Although there is no definitive therapy for Hunner's ulcer, at least we can make a diagnosis in these young women and explain their affliction to them, so that they can make the proper adjustments in their daily life and not be running from physician to physician for elucidation of their problem.

Although pollakiuria, urgency, and bladder discomfort are common in a number of diseases of the urinary tract, we believe that abnormal tenderness of the bladder floor to gentle palpation of the anterior lateral vaginal fornices suggests interstitial cystitis. The diagnosis is reinforced by the finding of a small-capacity, but otherwise normal, bladder on cystometry associated with urine specimens (catheterized) which contain many epithelial cells but no bacteria.

1405 E. Ann Street Ann Arbor, Michigan 48104

\section{References}

1. Prentiss, R. J., and Sorensen, M. B.: Female urology, in Karafin, L., and Kendall, R. A., Eds.: Urology, Lewis' Practice of Surgery, Hagerstown, Harper \& Row Publishers, Inc., vol. 2, chap. 18, 1973.

2. Smith, D. R.: General Urology, Los Altos, Lange Medical Publications, 1972, pp. 366-367.

3. Lowsley, O. S, and Kirwin, T. J.: Clinical Urology, Baltimore, Williams and Wilkins, Co., vol. 1, 1944, pp. 1013-1019. 